\title{
A Study on the Conversion Method Based on Standard Pedestrian Equivalent Factors at Signalized Crosswalks in China
}

\author{
Hui-ling Zhang ${ }^{1} \cdot$ Yuan-qiang Chen ${ }^{2} \cdot$ Xian-xian Pang ${ }^{1}$ \\ Received: 18 October 2021 / Revised: 16 December 2021 / Accepted: 27 January 2022 / Published online: 17 February 2022 \\ (c) The Author(s) 2022
}

\begin{abstract}
In order to more accurately assess the pedestrian level of service (PLOS) of crosswalks and to more rationally design the pedestrian signal timing, a method of calculating the standard pedestrian equivalent (SPE) factors at the signalized crosswalk in China was proposed in this study by considering the differences in static and dynamic characteristics amongst pedestrians. Firstly, the pedestrians were classified into five types using the pedestrian field data collected at the 3 signalized crosswalks in Chongqing, China. Then, by considering the parameters selection of the pedestrian LOS at signalized crosswalks, the SPE method based on pedestrian delays was derived, and the calculation models of pedestrian delays were presented for different pedestrian arrival patterns. A case analysis was conducted using the methods proposed in this paper, and the results show that the SPE values for minors, young and middle-aged females, elderly males and elderly females were 1.213, 1.067, 1.229 and 1.348, respectively. In addition, the results of the in-situ application to the pedestrian signal timing at the signalized crosswalk were achieved. This research can provide reference for practice related to transportation fields such as pedestrian capacity analysis and pedestrian signal timing design.
\end{abstract}

Keywords Signalized crosswalks $\cdot$ Standard pedestrian equivalent factor $\cdot$ Pedestrian delay $\cdot$ Mixed pedestrian flow $\cdot$ Pedestrian signal timing

\section{Introduction}

Rational calculation of the pedestrian flow is of a great significance in pedestrian timing analysis, crosswalk facility design, pedestrian level-of-service (PLOS) assessment and intersection traffic efficiency. The walking speed and occupancy areas differ from pedestrian types when they cross signalized crosswalks. The conversion analysis of different pedestrians allows for converting other types of pedestrians to a certain type of pedestrians by considering the differences. Thus, more reasonable pedestrian

Hui-ling Zhang

huilingz@126.com

Yuan-qiang Chen

542549927@qq.com

Xian-xian Pang

pxx24479@163.com

1 School of Traffic \& Transportation, Chongqing Jiaotong University, Chongqing 400074, China

2 Shenzhen New Land Tool Planning and Architectural Design Co., Ltd., Shenzhen 518172, China flow counting can be obtained, in comparison to ignoring the differences. Recently, many scholars have shifted their focus to the differences in dynamic and static characteristics of pedestrians. It was found that slow pedestrians or pedestrians with large vertical projection areas have a greater impact on road capacity [1]. In terms of the dynamic characteristics, many scholars have analyzed the differences and concluded that age and gender have significant effects on pedestrian crossing speed [2,3]. Sharifi found that the pedestrian average crossing speed would significantly reduce with disabilities on crowd [4]. Hediyeh et al. [5] found that pedestrian gait parameters were affected by crosswalk slope, pedestrian gender, age and group size based on computer vision technologies. In addition, slow pedestrians have received attentions during signal design $[6,7]$. With regard to the pedestrian occupancy characteristics, Fruin [8] believed that the thickness of the human body and the width of the shoulders were the primary considerations in the study of pedestrian space occupancy. Ye et al. [9] proposed the maximum width and maximum thickness of the design dimensions of the human body, and adopted the method of cluster sampling 
for a small sample size in order to measure the space occupancy of pedestrians according to their gender, age and whether they were carrying luggage etc.

With the population ageing, the pedestrian crossing behavior between the older pedestrian and youths are focused [10], and the methods to improve the safety of the elderly pedestrian were analyzed [11, 12].

All the above studies reveal the differences in static and dynamic characteristics of different crossing pedestrians. However, further analysis based on these differences is still necessary in order to provide more appropriate and reasonable data support for pedestrian crossing facility design, PLOS assessment and pedestrian flow statistics at signalized crosswalks. By analyzing the static space and dynamic characteristics of pedestrians, the pedestrian delay is considered as one of the core parameters for PLOS classification [9]. The study by Ding et al. [13] showed that pedestrians of different ages and genders showed different delays at the signalized crosswalk. To the best of our knowledge, apart from these studies, there is limited work focusing on static and dynamic differences of pedestrians when crossing the signalized crosswalk.

The follow structure of the paper are organized as follow: section 2 presented the pedestrian static and dynamic characteristics. The standard pedestrian and conversion method are explained in section 3. And a case is presented in section 4. Finally, the conclusion are summarized in section 5 .

In this paper, the space occupancy and the walking speed of the different pedestrians were analyzed, and a conversion method based on standard pedestrian equivalent (SPE) factors at the signalized crosswalks was proposed by taking the pedestrian delay as an input parameter. The static and dynamic differences of pedestrians at signalized crosswalks were then converted and analyzed. It may provide reference for pedestrian capacity analysis and pedestrian signal timing design.

\section{Analysis of Static and Dynamic Characteristics of Pedestrians}

In order to obtain the basic input data for the SPE conversion, the differences of static and dynamic characteristics among different types of pedestrians were firstly analyzed.

\subsection{Static Characteristics of Pedestrians}

To calculate pedestrian space occupancy, the 95th percentile design dimension is usually adopted as the design calculation criterion [7]. Thus, 95\% of the maximum shoulder width and chest thickness were used to reflect the space occupancy of standing pedestrians. Figure 1 shows the ellipse projection of the human body and illustrates the difference in shoulder width and chest thickness between adult males and minors $[14,15]$.

Table 1 shows the shoulder width of different types of pedestrians by reviewing the manuals and the literature in China $[14,15]$.

\subsection{Dynamic Characteristics of Pedestrians}

The differences in dynamic characteristics of walking pedestrians are mainly reflected in the walking speed [16], stride frequency and length [5]. The difference in stride length between two kinds of groups is shown in Fig. 2 . According to literature statistics [5], the average stride length of the young and middle-aged groups was $0.73 \mathrm{~m}$, and that of the minor group was $0.67 \mathrm{~m}$.

Table 1 Statistics of the maximum shoulder width of pedestrians

\begin{tabular}{llll}
\hline Parameter & \multicolumn{3}{l}{ Pedestrian types } \\
\cline { 2 - 4 } & Minors & Young \& middle-aged & Elderly \\
\hline $\begin{array}{l}\text { The maximum shoulder } \\
\text { width (m) }\end{array}$ & 0.392 & 0.469 & 0.409 \\
\hline
\end{tabular}

Fig. 1 Ellipse projection of human body (a) adult male (b) minor male

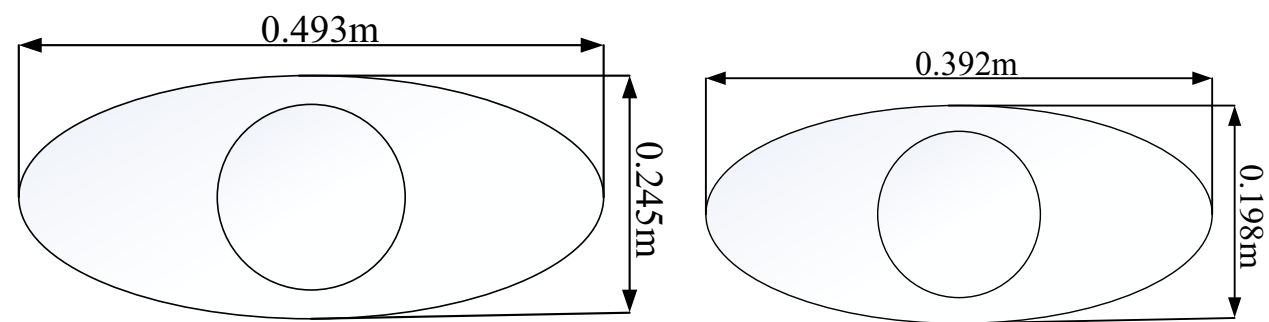

(a) adult male

(b) minor male 
Fig. 2 Comparison diagram of pedestrian stride length (a) Young and middle-aged male (b) Minor male

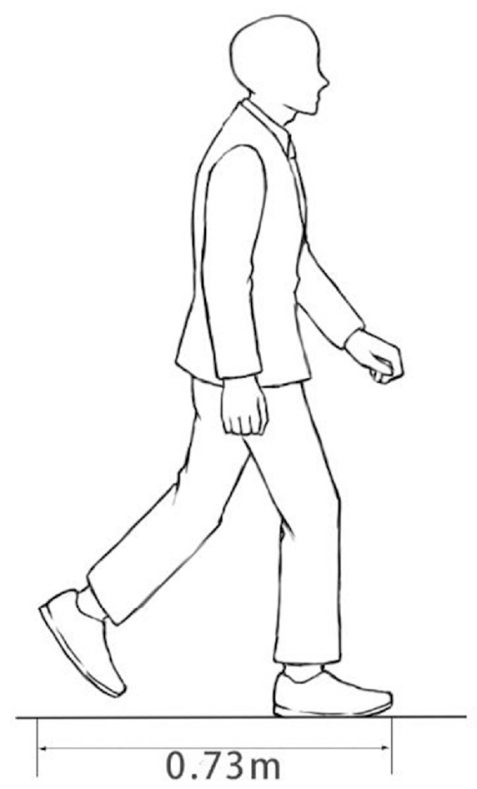

(a) Young and middle-aged male

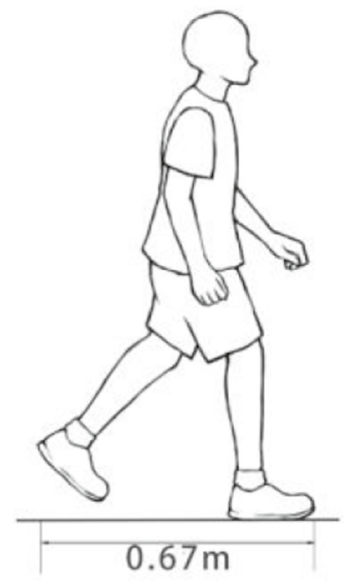

(b) Minor male
It can be seen from the relevant research that the relationship between walking speed, stride frequency and stride length can be expressed as [5].

$V=F \times S$

where $V$ is the walking speed of pedestrians $(\mathrm{m} / \mathrm{s}), F$ is the stride frequency (step/s) and $S$ is the stride length $(\mathrm{m} / \mathrm{step})$.

The changes of stride length and stride frequency can be reflected by walking speed. Thus, in order to simplify the calculation process, the walking speed was selected as the main parameter to demonstrate the dynamic characteristics of pedestrians. According to the relevant literature, there are significant differences in the walking speed of different types of pedestrians when crossing the street at signalized crosswalks, the children pedestrian group has the fastest speed and the elderly pedestrian group has the slowest speed [16].

\subsection{Pedestrian Classifications}

In order to investigate the rationality of pedestrian classification, the pedestrian crosswalks at Jinzi Street-Nanhu Road intersection, Nanhu Road-Qinglong Road intersection and Shuanglong Road-Huilong Road intersection in Chongqing, China were selected as the study sites through site visits and screening, and noted as Location A, B and C, respectively. The three survey sites had the following characteristics: (1) signal-controlled crosswalks; (2) no interference from leftturning or right-turning vehicles when the pedestrian green light was on; (3) the length of the crosswalks was larger than $12 \mathrm{~m}$ and the pedestrian crossing time was over $10 \mathrm{~s}$, which can accurately reflect the walking speed characteristics of pedestrians crossing the street and (4) a wide field of view for easy video recording. The specific locations and the conditions of these three crosswalks are shown in Fig. 3.

Table 2 shows the basic data of the sites collected by the field survey, including signal timing, crosswalk dimensions and traffic conditions.

Considering that the parameters related to pedestrian crossing manually collected under congested conditions were subject to errors for it was not easy to distinguish the collected pedestrians, the survey was conducted during the off-peak hours. In terms of the pedestrian sample, only the pedestrians who normally crossed the crosswalk during the green light were collected in the survey. Not including those who were disabled, those who diagonally crossed the street, ran or followed and these who were carrying heavy objects.

The pedestrian crossing speed can be determined through crosswalk length and pedestrian crossing time, expressed as

$V_{p}=\frac{B}{t}$

where $V_{\mathrm{p}}$ is pedestrian crossing speed $(\mathrm{m} / \mathrm{s}), t$ is the time for a single pedestrian to cross the crosswalk (s) and $B$ is the crosswalk length $(\mathrm{m})$.

A total of 842 samples of pedestrian walking speed were collected. To investigate the speed differences among pedestrians of different ages, pedestrians were divided into three groups, i.e., minors, elderly, young and middle-aged pedestrians. The Kruskal-Wallis ANOVA test was performed to examine the mean difference of the grouped data. The result 
Fig. 3 Selected study sites and detailed conditions at each location in Chongqing, China (a) The study sites (b) Location A (c) Location B (d) Location C

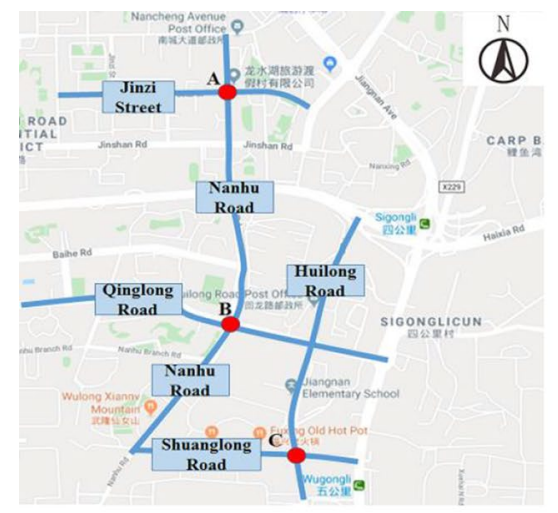

(a) The study sites

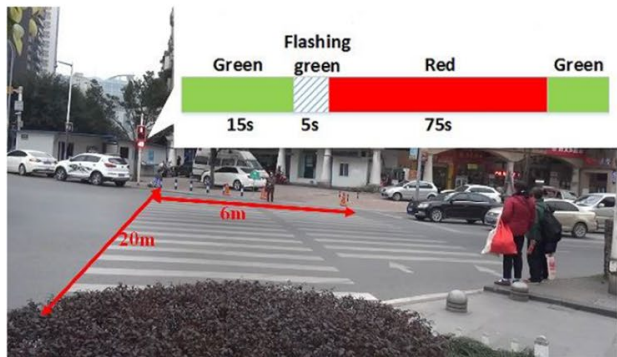

(c) Location B

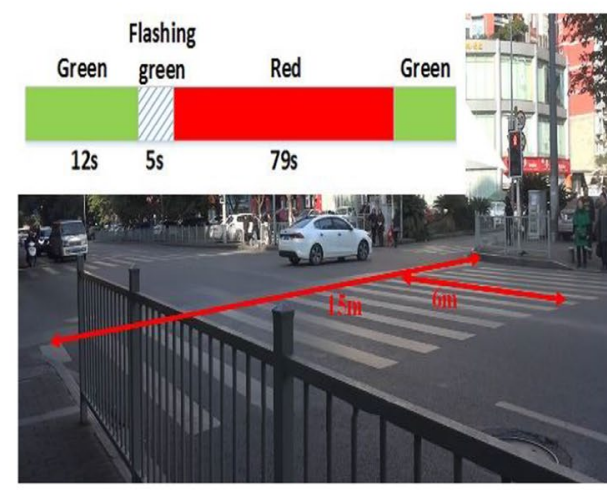

(b) Location A

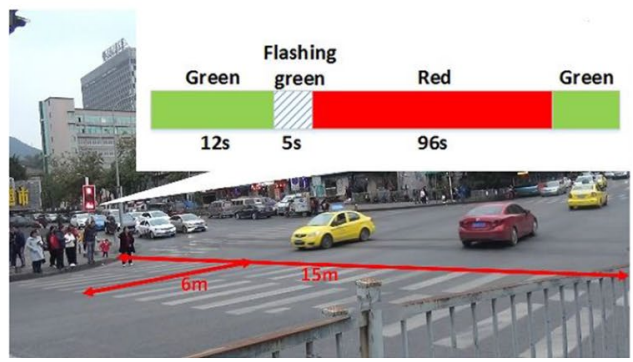

(d) Location C
Table 2 Specific conditions of study sites

\begin{tabular}{lllll}
\hline Parameter & Location & & \\
\cline { 3 - 5 } & & $\begin{array}{l}\text { Nanhu Road-Jinzi } \\
\text { Road intersection }\end{array}$ & $\begin{array}{l}\text { Nanhu Road- } \\
\text { Qinglong Road } \\
\text { intersection }\end{array}$ & $\begin{array}{l}\text { Nanhu Road- } \\
\text { Huilong Road } \\
\text { intersection }\end{array}$ \\
\hline Crosswalk code & A & B & C \\
Pedestrian volume (ped/h) & 138 & 511 & 196 \\
Traffic volume (veh/h) & & 1478 & 1107 & 1194 \\
Crosswalk width (m) & & 6 & 6 & 6 \\
Crosswalk length (m) & & 15 & 20 & 15 \\
Pedestrian safety island & & No & Yes & No \\
Pedestrian signal (s) & Green & 12 & 15 & 12 \\
& Flashing green & 5 & 5 & 5 \\
& Red & 79 & 75 & 96 \\
& Cycle time & 96 & 95 & 113 \\
\hline
\end{tabular}

shows that the overall test result of pedestrian crossing speed had a $p$ value of 0.000 , which was less than 0.05 . Thus, the null hypothesis should be rejected and this indicates that the difference in crossing speed among different types of pedestrians was statistically significant. For minors classified by gender, the speed differences were tested using Welch's ANOVA. The results did not show significant any differences (sig. $=0.168$ ), indicating that there was no significant difference between the walking speed of minor males and minor females. Therefore, minor males and minor females were combined into the same category of pedestrians. The same classification and validation were also performed for the young and middle-aged group and the elderly group by gender, which revealed significant differences between the genders. Thus, the pedestrians were finally classified into five categories, i.e., minors, young and middle-aged males, young and middle-aged females, elderly males and elderly females. The results are shown in Fig. 4.

Figure 4 shows that the young and middle-aged male group had the largest crossing speed with an average value of $1.266 \mathrm{~m} / \mathrm{s}$, while the elderly female group was the slowest with an average crossing speed of $0.955 \mathrm{~m} / \mathrm{s}$. 
Fig. 4 Speed distribution of different groups

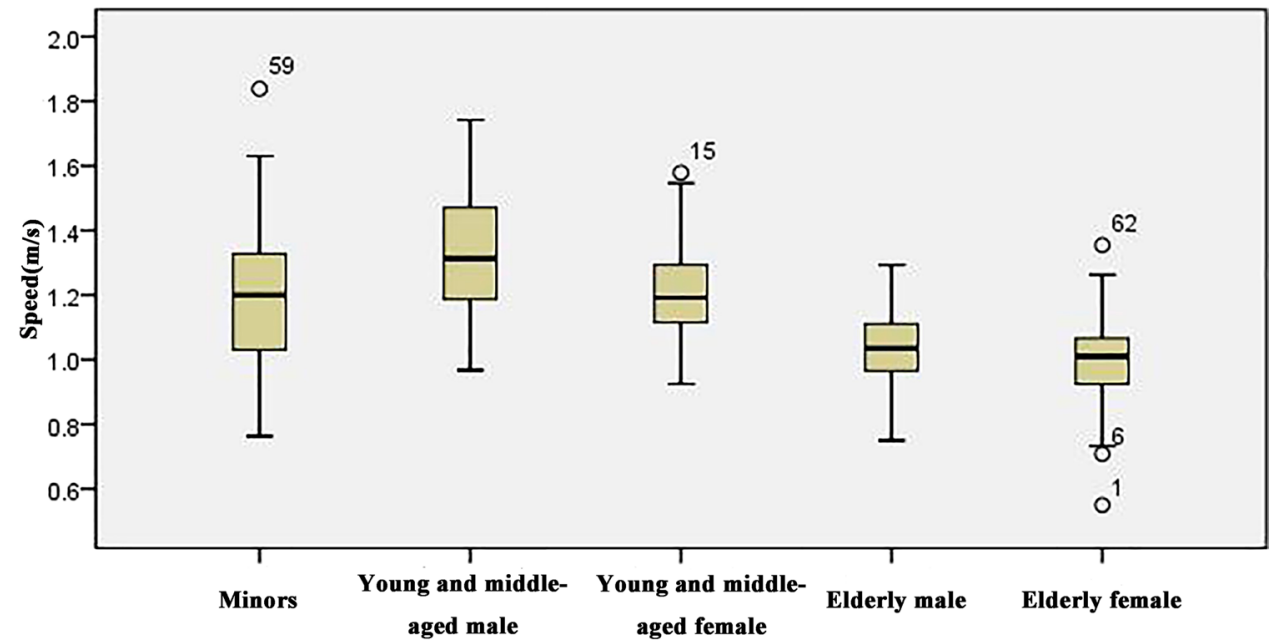

\section{Conversion Methodology}

\subsection{Selection of Standard Pedestrian and Conversion Method of Standard Pedestrian Equivalent Factor}

Referring to the definition of vehicle conversion method [7], the SPE factors are defined as the number of standard pedestrians of a specific type replacing individual pedestrians of other types under predominant conditions of road, traffic and control. The standard pedestrians are the types of pedestrians that make up the largest proportion in pedestrian traffic flow, referring to the standard vehicle. According to the demographic statistics of China in 2019 [17], young and middle-aged males were selected as the standard pedestrian in this research.

Delay is one of the core parameters in evaluating pedestrian crosswalk LOS and traffic efficiency at the signalized crosswalk [9]. Thus, the pedestrian delay was selected as the input parameter to calculate the SPE. Considering the relative methods [18], the relationship for the SPE factor based on pedestrian crossing delay under signal control conditions was established and expressed as

$S P E_{i j}=1+\frac{\Delta d_{i j}}{d_{0 j}}$

By considering pedestrian flow, Eq. (3) can be expressed as

$S P E_{i j}=1+\left(\frac{D_{t j}-D_{0 j}}{U_{i j}}\right) / d_{0 j}$

In addition, by considering the per-capita delay, Eq. (4) can be rewritten as
$S P E_{i j}=1+\frac{d_{t j}-d_{0 j}}{\omega_{i j} d_{0 j}}$

where $S P E_{i j}$ is the equivalent factor value of $i^{\text {th }}$ pedestrian type under $j^{\text {th }}$ service level, $\Delta d_{i j}$ is the additional delay caused by $i^{\text {th }}$ pedestrian type under $j^{\text {th }}$ service level (s), $d_{0 j}$ is the average delay of the standard pedestrian under $j^{\text {th }}$ service level (s), $D_{t j}$ is the total delay of the mixed pedestrian under $j^{\text {th }}$ service level (s), $D_{0 j}$ is the total delay of the standard pedestrian under $j^{\text {th }}$ service level (s), $d_{t j}$ is the average delay of the mixed pedestrian under $j^{\text {th }}$ service level (s), $\omega_{i j}$ is the percentage of $i^{\text {th }}$ pedestrian type mixed into the standard pedestrian under $j^{\text {th }}$ service level $\left(U / U_{i j}\right), U$ is the total pedestrian flow (ped/h) and $U_{i j}$ is the flow of $i^{\text {th }}$ pedestrian type under $j^{\text {th }}$ service level (ped/h).

\subsection{Distribution of Pedestrian Arrival}

Pedestrian delay calculation was based on the pedestrian arrival. Pedestrian arrival characteristics reflected pedestrian arrival patterns during a certain time interval and were generally described by Poisson and negative binomial distributions [19].

When the pedestrian flow was small, the interaction between the arriving pedestrians was weak. The sample variance was close to the sample mean and the pedestrian arrival obeyed the Poisson distribution. Thus, the probability $P(x)$ of $x$ pedestrians arriving in the counting time interval $t$ can be shown as

$P(x)=\frac{(\lambda t)^{x} e^{-\lambda t}}{x !}, x=0,1,2, \ldots, n$ 
where $\lambda$ is the average arrival rate per unit time(ped/s), $x$ is the number of arrivals(ped), $t$ is the counting interval(s) and $e$ is the base of the natural logarithm.

When the pedestrian flow was higher, pedestrian arrival showed larger fluctuations. The sample variance was larger than the sample mean, and the pedestrian arrival obeyed a negative binomial distribution. Thus, the probability $P(x)$ of $x$ pedestrians arriving in the counting time interval $t$ can be expressed as

$P(x)=C_{x+\beta-1}^{\beta-1} p^{\beta}(1-p)^{x}$

where $p$ is the model parameter $\left(p=m / S^{2}\right), \beta$ is the model parameter that should be an integer $\left(\beta=m^{2} /\left(S^{2}-m\right)\right), m$ is the sample mean and $S^{2}$ is the sample variance.

\subsection{Delay Calculation Models}

According to pedestrian arrival patterns, two delay models were presented. The first delay model can be used when the pedestrian arrival obeyed the Poisson distribution. The second delay model can be used when the arrival obeyed the negative binomial distribution.

\subsubsection{The First Delay Model}

During the pedestrian crossing signal cycle, when the pedestrian clearance signal turned on, pedestrians who were already in the crosswalk continued to pass. However, those who have arrived but haven not yet entered the crosswalk began to queue until the crosswalk signal turned green again. Then, the waiting pedestrians began to enter the crosswalk and cross. Due to crosswalk capacity restriction, all of the waiting pedestrians could not pass at once and needed to enter and pass through one after another, and later arriving pedestrians continued to queuing to pass. Pedestrians arriving after the queue has dispersed could directly cross the street until the pedestrian clearance signal of the next cycle turned on. At the signalized crosswalk, the relationship of arrival accumulation and dispersion of pedestrians crossing the crosswalk is shown in Fig. 5. For notations, $t_{i}$ is the pedestrian clearance time, $t_{r}$ is the red light time and $t_{s}$ is the dispersion time of queuing pedestrians.

In the model, it is assumed that (i) the pedestrian arrival characteristic obeyed the Poisson distribution, (ii) pedestrians crossed the crosswalk in rows, each row occupying the entire width of the crosswalk and (iii) pedestrians obeyed traffic rules.

The total number of pedestrians waiting to cross the street in a cycle can be calculated by

$q \times\left(t_{r}+t_{i}+t_{s}\right)=s \times t_{s}$

Furthermore, the dispersion time for the waiting pedestrians can be obtained by

$t_{s}=\frac{q \times\left(t_{r}+t_{i}\right)}{s-q}$

where $t_{s}$ is the dissipation time of pedestrians queuing (s), $q$ is pedestrian arrival rate (ped/s), $t_{r}$ is the red light time (s), $t_{i}$ is the pedestrian clearance time (s), $t_{i}=B / V_{p}, B$ is the crosswalk length $(\mathrm{m}), V_{p}$ is the pedestrians crossing speed $(\mathrm{m} / \mathrm{s})$, $s$ is pedestrian saturation flow rate (ped/s), $s=K /\left(L \cdot t_{q h}\right), K$ is the crosswalk width $(\mathrm{m}), L$ is the required lateral width for pedestrians (m), $t_{q h}$ is the time interval between two consecutive pedestrians (s) and, generally, $t_{q h}=1 \mathrm{~s}$.

The average pedestrian delay in a cycle can be obtained by $q \times C \times d=\frac{1}{2} q \times\left(t_{r}+t_{i}\right) \times\left(t_{r}+t_{i}+t_{s}\right)_{(10)}$

$d=\frac{\left(t_{r}+t_{i}\right)^{2} \times s}{2(s-q) \times C}$

where $d$ is the average pedestrian delay (s) and $C$ is the pedestrian signal cycle (s).
Fig. 5 Pedestrian crossing patterns without interference under signal control

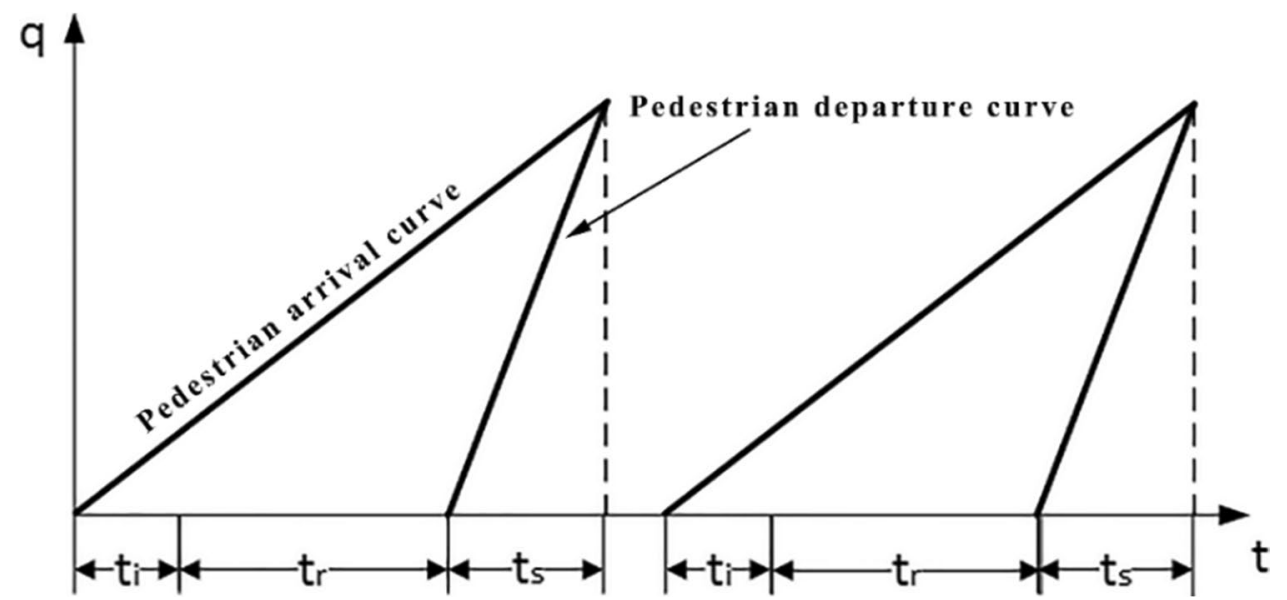


For the mixed pedestrian flow, the average speed $\left(V_{p}\right)$ of mixed pedestrians and the average lateral width $(L)$ were adopted to characterize the flow, expressed as

$V_{p}=\frac{n B}{\sum_{i=1}^{n} T_{i}}$

$L=\frac{\sum_{i=1}^{n} p_{i} L_{i}}{\sum_{i=1}^{n} p_{i}}$

where $n$ is the number of pedestrian types in the mixed pedestrian flow, $T_{i}$ is the average crossing time of the $i^{\text {th }}$ pedestrian type (s), $p_{i}$ is the flow of $i^{\text {th }}$ pedestrian type in the mixed pedestrian (ped/s), $L_{i}$ is the shoulder width of $i^{\text {th }}$ pedestrian type $(\mathrm{m})$.

\subsubsection{The second delay model}

Pedestrian delay included two components, i.e., the stopping delay and the crossing delay [20,21], and thus the average total delay $d$ is shown as

$d=d_{W T}+d_{C T}$

where $d$ is the average delay under signal control (s), $d_{W T}$ is the delay due to waiting for the green light i.e., stopping delay (s), and $d_{C T}$ is the difference between the actual crossing time and the design crossing time,i.e., the crossing delay (s).

According to the study by Nagraj and Vedagiri [22], the calculation model of $d_{W T}$ is represented as

$d_{W T}=K_{c} K_{N U} * \frac{(C-G)^{2}}{2 * C}$

where $K_{N U}$ can be calculated by [19].

$K_{N U}=\frac{C *\left(n_{t}-n_{g}\right)}{n_{t} *(C-G)}$

where $K_{c}$ is the correction factor for compliance with the signal control, i.e., the percentage of pedestrians arriving during the red light phase and complying with the signal control, $K_{N U}$ is the non-uniform arrival rate, $C$ is the pedestrian signal cycle (s), $G$ is the green light time (s), $n_{t}$ is the total number of pedestrians (ped) and $n_{g}$ is the number of pedestrians arriving during the green phase (ped).

The ideal crossing time was determined by the crosswalk length and the ideal crossing speed, which was taken from the average crossing speed of some randomly selected pedestrian samples that were not significantly disturbed by vehicles [21]. Thus, the crossing delay $d_{C T}$ is expressed as
$d_{C T}=\frac{\sum_{i=1}^{n} T_{i}^{\prime}}{n}-\frac{B}{\frac{\sum_{j=1}^{m} V_{j}}{m}}=\frac{\sum_{i=1}^{n} \frac{B}{V_{i}}}{n}-\frac{m B}{\sum_{j=1}^{m} V_{j}}$

where $\frac{\sum_{i=1}^{n} T_{i}^{\prime}}{n}$ is average actual pedestrian crossing time (s), $T_{i}{ }^{\prime}$ is the crossing time of the $i^{\text {th }}$ pedestrian in the mixed pedestrian flow (s), $V_{i}$ is the crossing speed of the $i^{\text {th }}$ pedestrian in the mixed pedestrian flow $(\mathrm{m} / \mathrm{s})(i=1,2, \ldots, n), n$ is the total number of mixed pedestrians crossing the street in a signal cycle, $\frac{B}{\frac{\sum_{j=1}^{m} v_{j}}{m}}$ is the average ideal crossing time (s) and $V_{j}$ is the crossing speed of $j^{\text {th }}$ pedestrian randomly selected from pedestrian samples that were not significantly disturbed by traffic flow $(\mathrm{m} / \mathrm{s})(j=1,2, \ldots, m), m$ is the number of randomly selected samples.

When $d_{C T} \leq 0$, it indicates that there was no delay for pedestrians crossing the street. When $d_{C T} \geq 0$, the value of $d_{C T}$ represented the crossing delay.

\section{Case Analysis}

Considering the large population and the larger number of crossing pedestrians in China, the method in this paper was validated by taking the observed data of Nanhu Road and Qinglong Road intersection as an example. The average crossing speed of 5 types of collected pedestrian samples is shown in Table 3.

The histogram of pedestrian arrival frequency with a sampling interval of $5 \mathrm{~s}$ is shown in Fig. 6.

\subsection{Distribution Test of Pedestrian Arrival}

The descriptive analysis results of the data from the pedestrian arrival survey are presented in Table 4.

Then, a negative binomial distribution model of pedestrian arrival was established and subsequently tested for goodness of fit using the Chi-squared test. According to Table 4, the mean and the standard deviation are not equal, the negative binomial distribution model of pedestrian

Table 3 Pedestrian crossing speed at crosswalks

\begin{tabular}{llllll}
\hline Parameter & \multicolumn{2}{l}{ Pedestrian } & & \\
\cline { 2 - 6 } & Minors & $\begin{array}{l}\text { Young and } \\
\text { middle- } \\
\text { aged males }\end{array}$ & $\begin{array}{l}\text { Young and } \\
\text { middle- } \\
\text { aged } \\
\text { females }\end{array}$ & $\begin{array}{l}\text { Elderly } \\
\text { males }\end{array}$ & $\begin{array}{l}\text { Elderly } \\
\text { females }\end{array}$ \\
\hline $\begin{array}{l}\text { Average } \\
\text { pedes- } \\
\text { trian } \\
\text { speed } \\
\left(\mathrm{m} \cdot \mathrm{s}^{-1}\right)\end{array}$ & 1.155 & 1.266 & 1.156 & 1.031 & 0.995 \\
\hline
\end{tabular}


Fig. 6 Histogram of pedestrian arrival frequency

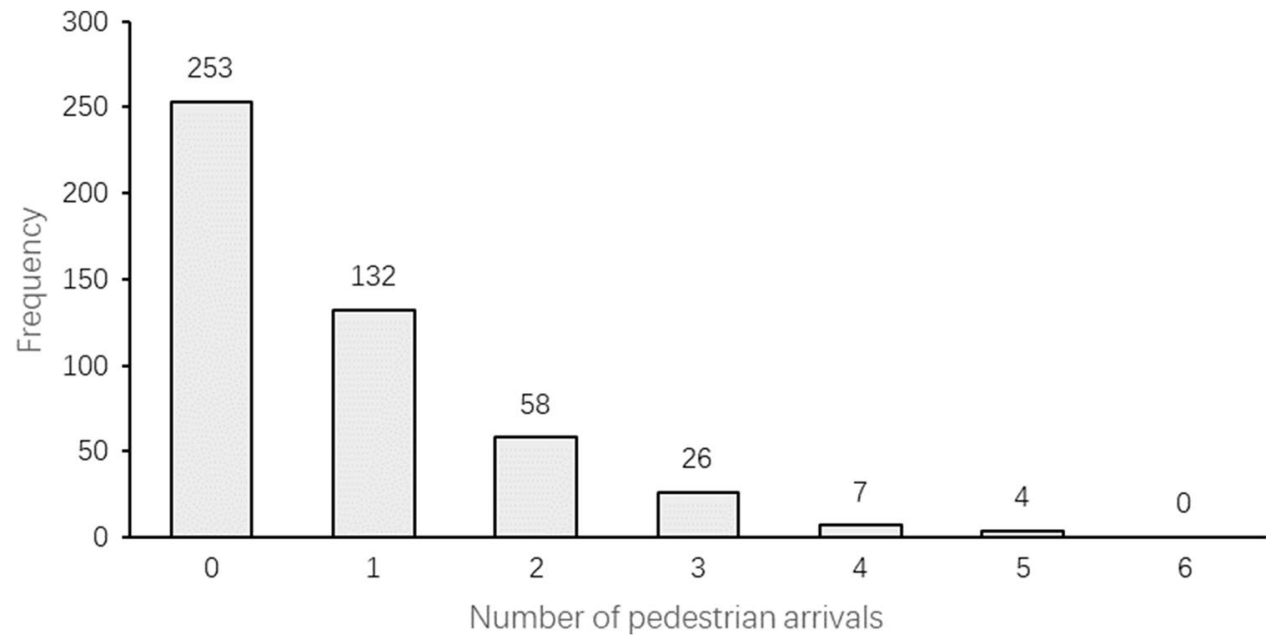

Table 4 Pedestrian arrival frequency description statistics

\begin{tabular}{llllll}
\hline $\mathrm{N}$ & Minimum & Maximum & Mean & $\begin{array}{l}\text { Std. } \\
\text { Deviation }\end{array}$ & Variance \\
\hline 480 & 0 & 5 & 0.78 & 1.041 & 1.083 \\
\hline
\end{tabular}

arrival can be applied and the results of Chi-squared tests are shown in Table 5.

Table 5 shows that when the confidence interval was set as $95 \%$, the value of $\chi^{2}$ of pedestrian arrival was smaller than $\chi_{d}^{2}$, indicating that the pedestrian arrival obeyed the negative binomial distribution.

\subsection{Conversion Results}

From the analysis in Section 4.1, the pedestrian arrival at the verified intersection obeyed a negative binomial distribution. Thus, the second delay model was used to calculate the pedestrian delay. The results of average pedestrian delay of both mixed and standard pedestrian flows and the SPE factors are shown in Table 6.

\subsection{Application and Analysis}

To analyze the influence of the pedestrian conversion factor proposed in this paper on the pedestrian crossing demand and the crosswalk signal timing, the pedestrian crossing time was calculated by the relevant equation listed by HCM2010 using the case study results as the input [7], thus:

$g t=\left\{\begin{array}{l}3.2+0.81 \frac{N_{p}}{W_{e}}+\frac{B}{V_{a}}, W_{e} \geq 3.0 \mathrm{~m} ; \\ 3.2+0.27 N_{p}+\frac{B}{V_{a}}, W_{e}<3.0 \mathrm{~m},\end{array}\right.$

where $g_{t}$ is the pedestrian crossing time (s), $N_{p}$ is the number of pedestrians required to cross the street within a signal cycle (ped), $W_{e}$ is the effective width of the crosswalk (m) and $V_{a}$ is the average pedestrian crossing speed $(\mathrm{m} / \mathrm{s})$..

The survey results of the Nanhu Road-Qinglong Road crosswalk was adopted as the basis, and it is assumed that the total number of pedestrians who need to cross the street in a signal cycle was 60 , among which the number of minors, young and middle-aged males, young and middleaged females, elderly males and elderly females were 12 , respectively. The pedestrian crossing demand was 2274
Table 5 The results of Chisquared tests

Table 6 Average delay and SPE factors for pedestrians

\begin{tabular}{lllllll}
\hline Mean & Variance & $p$ & $\beta$ & $\chi^{2}$ & $\chi_{d}^{2}$ & $\mathrm{~N}$ \\
\hline 0.78 & 1.083 & 0.720 & 2 & 1.579 & 5.992 & 480 \\
\hline
\end{tabular}

\begin{tabular}{|c|c|c|c|c|c|}
\hline \multirow[t]{2}{*}{ Parameter } & \multicolumn{5}{|c|}{ Pedestrian } \\
\hline & Minors & $\begin{array}{l}\text { Young and } \\
\text { middle-aged } \\
\text { males }\end{array}$ & $\begin{array}{l}\text { Young and } \\
\text { middle-aged } \\
\text { females }\end{array}$ & Elderly males & Elderly females \\
\hline Average delay (s) & 19.651 & 18.848 & 19.100 & 19.710 & 20.160 \\
\hline SPE & 1.213 & 1.000 & 1.067 & 1.229 & 1.348 \\
\hline
\end{tabular}


$\mathrm{ped} / \mathrm{h}$ when the SPE factor was not considered, and 2551 $\mathrm{ped} / \mathrm{h}^{*}$ SPE when the factor was incorporated. For signal timing, based on Eq. (20), the green time for each cycle of the crosswalk was $28 \mathrm{~s}$ without conversion, and should be $30 \mathrm{~s}$ after considering conversion.

\section{Conclusions}

Different kind of pedestrian have different body size and space occupancy, the young and middle age people have large shoulder wide and this group pedestrian needs wider space on the crosswalk. Moreover, as many analyzing results showed the different kind of pedestrian has different crossing speed $[2,4]$. Generally, the young and middle-aged group had the highest crossing speed. According to field survey data, pedestrians were divided into five groups in this paper, including minors, young and middle-aged males, young and middle-aged females, elderly males and elderly females. Then, the static and dynamic differences of these five types of pedestrians during crossing the signal-controlled crosswalk were analyzed, and the classification rationality was proved. Using the field survey data, it is tested the young and middle-aged group with an average value of $1.266 \mathrm{~m} / \mathrm{s}$, while the elderly female group was the slowest with an average speed of $0.955 \mathrm{~m} / \mathrm{s}$. For the delay pedestrian is one of the core parameters to evaluate the PLOS, so a delaybased model for calculating pedestrian conversion factors at signalized crosswalks was proposed in this paper. But the pedestrian delay is difficult to get directly, so two kind of delay calculation models were deduced considering the pedestrian's arriving type. Lastly, a case study is calculated in order to compare with the method without considering the SPE factors, and it is proved the pedestrian crossing demand is larger than the condition that not using the SPE method because the elderly pedestrian has the slowly crossing speed and the SPE factors are 1.229 and 1.348 for the male and female elderly pedestrians. Same as the pedestrian crossing demand, the pedestrian signal time is longer than the time that not considering the pedestrian crossing difference. So the method incorporating the SPE factors can better reflect the influence of different types of pedestrians on pedestrian flow and improve the accuracy of signal timing. This research will help to enrich the SPE factor calculation method, and also provides a more accurate basis for the assessment of pedestrian flow operation status of pedestrian crossing facilities and crossing facility design.

Since the data and the experiments were both based on real scenarios and it is difficult to get every PLOS scene to test the factors sensibility, it needs more data and more scenarios to validate the factors to use it in more wide condition. Moreover the pedestrian delay models should be improved according to the real pedestrian arrival type.
Acknowledgements The research is supported by Research Supervisor Team Building Foundation of Chongqing, China (JDDSTD2018007), Project of Innovative Research Groups for Universities in Chongqing (Grant No.CXQT21022), the Natural Science Foundation of Chongqing (cstc2020jcyj- msxmX0083), and the Science and Technology Research Program of Chongqing Municipal Education Commission (KJQN201800727).

\section{Declarations}

Conflict of Interest The authors declare that we have no conflict of interest.

Open Access This article is licensed under a Creative Commons Attribution 4.0 International License, which permits use, sharing, adaptation, distribution and reproduction in any medium or format, as long as you give appropriate credit to the original author(s) and the source, provide a link to the Creative Commons licence, and indicate if changes were made. The images or other third party material in this article are included in the article's Creative Commons licence, unless indicated otherwise in a credit line to the material. If material is not included in the article's Creative Commons licence and your intended use is not permitted by statutory regulation or exceeds the permitted use, you will need to obtain permission directly from the copyright holder. To view a copy of this licence, visit http://creativecommons.org/licenses/by/4.0/.

\section{References}

1. Galiza, R.J., Ferreira, L.: Developing standard pedestrian equivalent (SPE) factors: a pce approach for dealing with pedestrian diversity. Transp. Res. Rec.: J. Transp. Res. Board. 2299, 166-173 (2012)

2. Tarawneh, M.S.: Evaluation of pedestrian speed in Jordan with investigation of some contributing factors. J. Saf. Res. 32(2), 229-236 (2001)

3. Marisamynathan, S., Vedagiri, P.: Modeling pedestrian level of service at signalized intersection under mixed traffic conditions. Transp. Res. Rec.:J. Transp. Res. Board. 2634, 1-18 (2017)

4. Sharifi, M.S., Stuart, D.: Christensen KM, et al analysis of walking speeds involving individuals with disabilities in different indoor walking environments. J. Urban Plan. Dev. 142(1), 04015010 (2016). https://doi.org/10.1061/(asce)up.1943-5444.0000288

5. Hediyeh, H., Sayed, T., Zaki, M.H., et al.: Pedestrian gait analysis using automated computer vision techniques. Transp. A: Transp. Sci. 10(3), 214-232 (2014)

6. Gates, T.J., Noyce, D.A., Bill, A.R., et al.: Recommended walking speeds for timing of pedestrian clearance intervals based on pedestrian characteristics of the pedestrian population. Transp. Res. Rec.: J. Transp. Res. Board. 38-47 (2006)

7. Transportation Research Board: Highway Capacity Manual 2010. TRB, National Research Council, Washington DC (2010)

8. Fruin, J.J.: Pedestrian planning and design [M]. Metropolitan Association of Urban Designers \& Environmental Planners, (1971).

9. Ye, J.H., Chen, X.H., Liu, Y.: Human dimensions for pedestrian traffic design. J. Wuhan Univ. Technol.: Transp. Sci. Eng. 34(1), 10-14 (2009)

10. Dommes, A.: Street-crossing workload in young and older pedestrians. Accid. Anal. Prev. 128, 175-184 (2019)

11. Das, S., Bibeka, A., Sun, X.D., et al.: Elderly pedestrian fatal crash-related contributing factors applying empirical Bayes 
geometric mean method. Transp. Res. Rec. 2673(08), 254-263 (2019)

12. Nakagawa, Y.: Elderly pedestrians' self-regulation failures and crash involvement: the development of typologies. Accid. Anal. Prev. 133, 1-13 (2019)

13. Ding, T.Q., Li, W., Xi, J.F., et al.: Analysis of pedestrian safety at signalized intersections based on pedestrian crossing behavior[C]//the 14th COTA international conference of transportation professionals, Changsha. China. 2291-2303 (2014). https://doi.org/10.1061/9780784413623.220

14. State Bureau of Technical Supervision. Chinese adult body size: GB/T 10000-1988[S].Beijing: China standard press, (1989).

15. National technical committee on ergonomics standardization (SAC/TC7). GB/T 26158-2010, Human dimension of Chinese minors[S]. Beijing: China standard press, (2011).

16. Goh, B.H., Subramaniam, K., Wai, Y.T.: Pedestrian crossing speed: the case of Malaysia. Int. J. Traffic Transp. Eng. 2(4), 323-332 (2012)

17. Department of Economic and Social Affairs: World Population Prospects: the 2019 Revision. United Nations, New York (2019)

18. Benekohal, R.F., Zhao, W.X.: Delay-based passenger car equivalents for trucks at signalized intersections. Transp. Res. A. 34, 437-457 (2000). https://doi.org/10.1016/s0965-8564(99)00026-9
19. Qu, S.W., Zhou, L.J., Wang, D.H.: Bicycle and pedestrians' arrival and departure characteristics at signalized intersection. J. Highw. Transp. Res. Dev. 21(8), 91-94 (2004) (in Chinese)

20. Virkler, M.: Pedestrian compliance effects on signal delay. Transp. Res. Rec. 1636, 88-91 (1994). https://doi.org/10.3141/1636-14

21. Li, Q.F., Wang, Z.A., Yang, J.G.: Pedestrian delay estimation at signalized intersections in developing cities. Transp. Res. A. 39(1), 61-73 (2005). https://doi.org/10.1016/j.tra.2004.11.002

22. Nagraj, R., Vedagiri, P.: Modeling pedestrian delay and level of service at signalized intersection crosswalks under mixed traffic conditions. Transp. Res. Rec.: J. Transp. Res. Board. 2394, 70-76 (2013). https://doi.org/10.3141/2394-09

Publisher's Note Springer Nature remains neutral with regard to jurisdictional claims in published maps and institutional affiliations. 